\title{
Hipertricosis cubital en una paciente con pubarquia precoz. Caso clínico
}

\author{
Hypertrichosis cubiti in a girl with precocious puberty. Case report
}

\author{
Dr. J. David Herrero-Morina, Dra. Nuria Fernández González ${ }^{b}$, Lic. Cruz Gutiérrez Diéza, \\ Dra. Cristina Rodríguez Dehlic y Dra. Cristina Molinos Norniella ${ }^{d}$
}

\begin{abstract}
RESUMEN
Lahipertricosis cubital es un aumento localizado dela densidad, longitud y espesor del vello. Es una entidad benigna con muy escasos pacientes descritos en la literatura médica (alrededor de medio centenar). La mitad de los casos descritos asocian otros defectos o malformaciones, y la otra mitad son problemas puramente estéticos. La pubarquia precoz en niñas se define como el inicio del vello púbico antes de los 8 años de edad. Se presenta a una paciente de 6 años con la asociación no descrita previamente de hipertricosis cubital y pubarquia precoz.

Palabras clave: hipertricosis cubital, sindrome del codo piloso, pubarquia precoz.
\end{abstract}

\begin{abstract}
Hypertrichosis cubiti is a localized increase in hair density, length and thickness. It is an uncommon and benign entity with very few patients described in the medical literature (more or less than half a hundred). Half of the described patients associate other defects or malformations and the other half are purely aesthetic cases. Early pubarche in girls is defined as the onset of pubic hair before 8 years of age. We present a sixyear-old patient with the association not previously described of hypertrichosis cubiti and precocious pubarche.

Key words: hypertrichosis cubiti, hairy elbow syndrome, premature pubarche.
\end{abstract}

http: / / dx.doi.org/10.5546/ aap.2018.e765

Cómo citar: Herrero-Morín D, Fernández González N, Gutiérrez Díez C, Rodríguez Delhi C, et al. Hipertricosis cubital en una paciente con pubarquia precoz. Caso clínico. Arch Argent Pediatr 2018;116(6):e765-e768.

a. Centro de Salud de Infiesto, Área Sanitaria VI, Asturias.

b. Hospital Universitario Central de Asturias, Oviedo, Asturias.

c. Hospital San Agustín, Avilés, Asturias.

d. Hospital de Cabueñes, Gijón, Asturias.

España.

Correspondencia:

Dr. J. David Herrero-Morín: josedahm@yahoo.es

Financiamiento: Ninguno.

Conflicto de intereses: Ninguno que declarar.

Recibido: 2-3-2018

Aceptado: 14-6-2018

\section{INTRODUCCIÓN}

La hipertricosis cubital se define como el aumento localizado de la densidad, longitud y espesor del vello. Es una entidad descrita por primera vez hace 40 años y poco publicada en la literatura médica. La mitad de los casos descritos tienen talla baja y otras malformaciones asociadas; en el resto, el hallazgo es aislado y con repercusión exclusivamente estética.

\section{CASO CLÍNICO}

Se presenta a una niña de 6,0 años de edad que acudió a la consulta de atención primaria por una patología común. Su peso era de 21,3 $\mathrm{kg},-0,21$ desviaciones estándar (DE), talla de $121 \mathrm{~cm},+0,84 \mathrm{DE}$ (tablas de Estudios Españoles de Crecimiento de 2010, Carrascosa et al.) y la tensión arterial (TA) de 98/68 mmHg (+0,13/+0,98 DE). La velocidad de crecimiento (talla previa registrada 2 años antes) era de -0,22 DE. El fenotipo de la paciente era normal, sin rasgos faciales u otros sugerentes de síndrome genético. En la exploración, se destacó cabello rubio y liso, con hipertricosis cubital evidente (vello terminal grueso y rubio de $4 \mathrm{~cm}$ en la superficie extensora de los codos; Figura 1), junto con pubarquia de vello oscuro, grueso y liso de 2-3 cm en los labios mayores, sin inicio de botón mamario ni vello axilar (estadio de Tanner P2 M1); los genitales eran femeninos sin otras alteraciones. Rehistoriada, la pubarquia se inició a los 5 años, sin cambios en el olor corporal y no se encontraron contactos con disruptores endócrinos, salvo el consumo esporádico de carne procesada. Como antecedentes personales, nació fruto de una gestación a término y con peso adecuado a la edad gestacional, y su desarrollo psicomotor fue normal. En la historia familiar, la madre presentaba hipertricosis cubital desde la infancia (menos intensa en la edad adulta), con desarrollo puberal normal. El hermano presentó, desde la infancia, hipertricosis en las extremidades inferiores, no en el resto. La talla de la madre era 169 cm, y la del padre, 
$170 \mathrm{~cm}$; no había consanguinidad entre ambos. Se realizó un estudio hormonal a la paciente con perfil tiroideo y niveles basales de sulfato de dehidroepiandrosterona, androstendiona, testosterona y 17-OH-progesterona, que resultaron normales (Tabla 1). La edad ósea fue de 5 años y 9 meses (Atlas, de Gilsanz y Ratib), y la ecografía pélvica prepuberal y con región suprarrenal normal. Se programaron controles clínicos cada, aproximadamente, seis meses, y, en el primero realizado, no se apreciaron cambios en el estadio puberal de Tanner ni aceleración de la velocidad de crecimiento.

\section{DISCUSIÓN}

La hipertricosis cubital (síndrome del codo piloso) es una entidad rara o, mucho más probable, infradiagnosticada. Fue descrita por primera vez por Beighton en 1979, que sugirió, ya en ese momento, una base etiológica genética. ${ }^{1}$ Desde entonces, se han descrito alrededor de 40 casos; $^{2}$ la mayoría, esporádicos, pero también algunos con agrupación familiar que hacen pensar en una herencia recesiva o dominante con penetrancia incompleta y expresividad variable. Aunque la mayoría de los autores han referido casos aislados, esta entidad ha sido descrita también asociada a síndromes genéticos. Incluso

FIGURA 1. Imagen de la hipertricosis en la zona extensora del codo

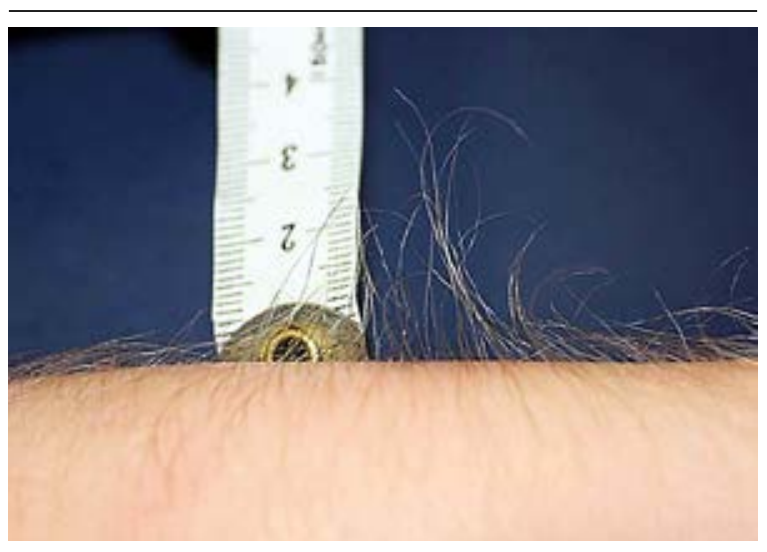

un autor ha reportado hasta 5 pacientes con esta entidad asociada al síndrome de WiedemannSteiner. ${ }^{3}$

La mitad de las hipertricosis cubitales descritas se presentan como manifestación aislada, en cuyo caso se trata de un proceso puramente estético que alcanza su máxima expresión a los 5 años y remite, en la mayor parte de los casos, en la adolescencia, por lo que no precisan estudios complementarios de ningún tipo y se procura un manejo conservador en espera de su resolución. La otra mitad asocian talla baja, y son estos los casos en que, habitualmente, se han descrito otras manifestaciones, como dismorfia facial, alteraciones psicomotoras y del lenguaje o anormalidades esqueléticas (Tabla 2), aunque la relación con la hipertricosis es discutida. ${ }^{4,5}$

La benignidad del proceso en los casos sin manifestaciones asociadas hace improbable que las familias consulten por este motivo $y$, más aún, que sea reconocido como una patología por el profesional sanitario o que decida su comunicación científica, con lo que es posible que esta entidad sea mucho más frecuente de lo esperable por los escasos casos reportados. Sirve de ejemplo nuestro caso, en que la consulta inicial no estuvo motivada por el vello anormal en los codos (curiosamente, tampoco por el inicio precoz de vello púbico antes de los 8 años en niñas o pubarquia precoz). ${ }^{6}$ Igualmente, aunque la mitad de los casos descritos tengan otras manifestaciones asociadas, cabe esperar un muy probable sesgo que lleva a publicar los casos peculiares, lo que puede hacer que solo un mínimo número de casos asocien talla baja u otras malformaciones que podrían, o muchas de ellas probablemente no, estar asociadas con esta entidad. En nuestro caso, la asociación de hipertricosis cubital con pubarquia precoz no relacionada con sobrecarga androgénica, con una talla normal, al no tener constancia de que haya sido publicado previamente en la literatura médica, es lo que ha motivado esta presentación, aun siendo conscientes de la probabilidad de asociación casual.

TABLA 1. Niveles basales de 17-OH-progesterona y andrógenos en la paciente

\begin{tabular}{lcl}
\hline 17-OH-progesterona & $0,75 \mathrm{ng} / \mathrm{ml}$ & Rango de normalidad de 1-9 años: 0,16-1,02 ng/ml \\
Sulfato de dehidroepiandrosterona & $0,19 \mathrm{mcg} / \mathrm{ml}$ & Rango de normalidad de 1-9 años: 0,10-1,86 ng/ml \\
Androstendiona & $<0,3 \mathrm{ng} / \mathrm{ml}$ & Rango de normalidad en la infancia: hasta 0,5 ng/ml \\
Testosterona & $<0,2 \mathrm{ng} / \mathrm{ml}$ & Rango de normalidad en la infancia: hasta 0,3 ng $/ \mathrm{ml}$ \\
\hline
\end{tabular}


Presentación de casos clínicos / Arch Argent Pediatr 2018;116(6):e765-e768 / e767

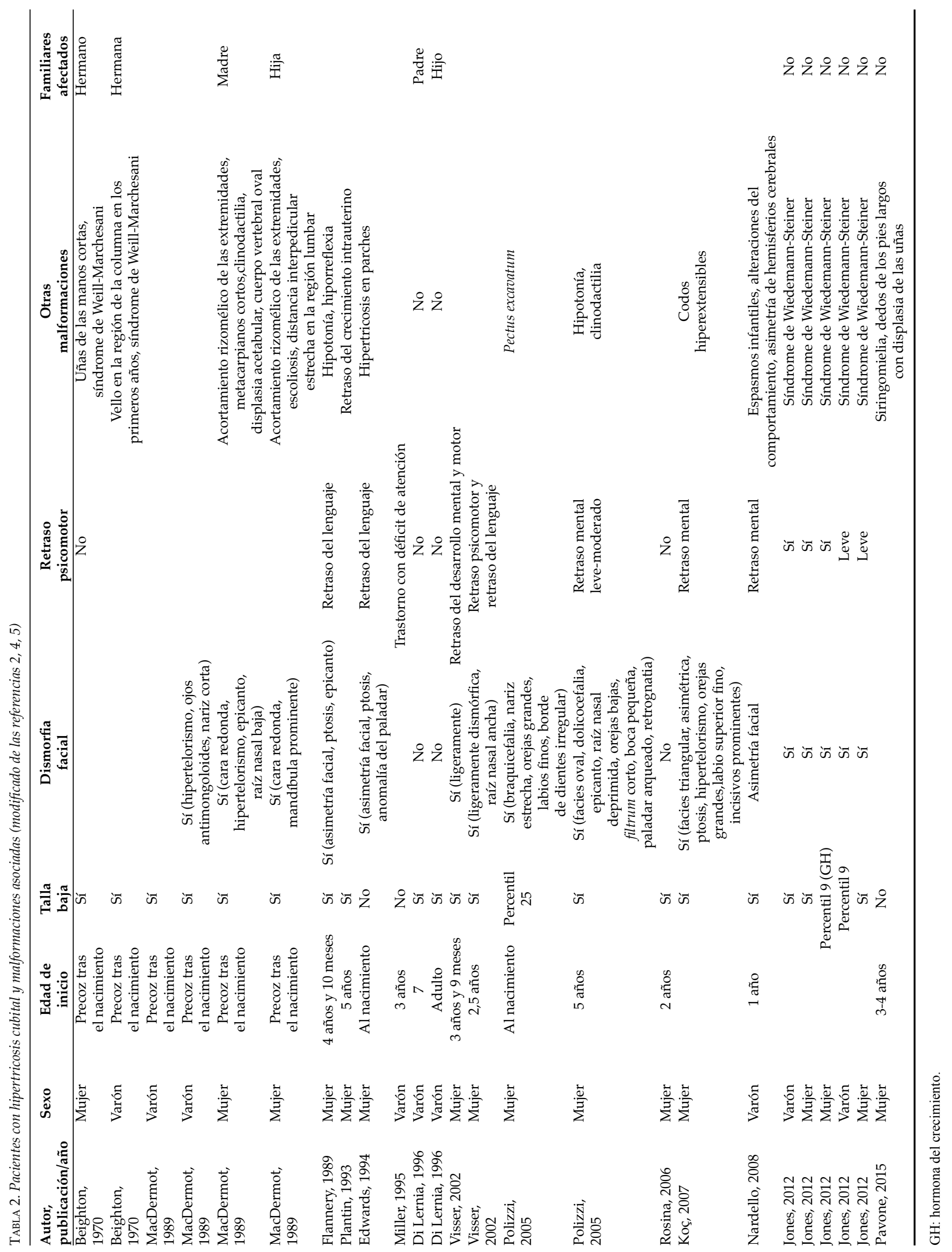


Aunque, en muchos casos presentados, incluso sin otras manifestaciones asociadas, se llevaron a cabo estudios complementarios, la benignidad de esta entidad no lo indica. ${ }^{5}$ En el nuestro, fue la pubarquia precoz lo que nos llevó a realizar estudios para descartar una hiperplasia adrenal congénita no clásica u otra entidad que justificara el aumento del vello como consecuencia del aumento de andrógenos. ${ }^{6}$ Dada la normalidad de los estudios iniciales, se informó a la familia de la evolución esperable de ambos procesos y se recomendaron medidas conservadoras para la hipertricosis $\mathrm{s}^{7,8}$ y seguimiento periódico del crecimiento y del estadio puberal.

Como conclusión, a pesar de su escasa frecuencia, es importante que el pediatra conozca el cuadro de hipertricosis cubital y su benignidad en los casos aislados, así como la importancia del seguimiento periódico, al menos, hasta la pubertad, más aún en casos como el descrito, en el que es preciso comprobar que el desarrollo puberal definitivo se produce a edad adecuada.

\section{REFERENCIAS}

1. Beighton P. Familial hypertrichosis cubiti: hairy elbows syndrome. J Med Genet 1970; 7(2):158-60.

2. Pavone V, Testa G, Falsaperla R, Sessa G. Syringomyelia and bone malformations in the setting of hypertrichosis cubiti (hairy elbow syndrome). J Orthop Case Rep 2015; 5(2):32-4.

3. Jones WD, Dafou D, McEntagart M, et al. Denovo mutations in MLL cause Wiedemann-Steiner syndrome. Am J hum Genet 2012; 91(2):358-64.

4. Tng VE, de Zwaan S. Hypertrichosis cubiti, a case report and literature review. Clin Case Rep 2015; 4(2):138-42.

5. Herrero-Morín JD, Calvo Gómez-Rodulfo A, García López E, et al. Hipertricosis cubital o síndrome del codo piloso. Acta Pediatr Esp 2010; 68(10):515-7.

6. Alonso M, Ezquieta B. Hiperplasia suprarrenal congénita no clásica o tardía. Rev Esp Endocrinol Pediatr 2012; 3(Suppl):61-73.

7. Vashi RA, Mancini AJ, Paller AS, Primary generalized and localized hypertrichosis in children. Arch Dermatol 2001; 137(7):877-84.

8. MacDermot KD, Patton MA, Williams MJ, Winter RM. Hypertrichosis cubiti (hairy elbows) and short stature: a recognisable association. J Med Genet 1989; 26(6):382-5. 